\title{
Copépodos Notodiaptomus sp. Kiefer (Crustacea, Calanoida) naturalmente infectados com metacestódeos no reservatório do Juqueri, São Paulo, Brasil
}

\author{
Rubens Riscala Madi ${ }^{1,4}$, Marlene Tiduko Ueta ${ }^{1}$, Tarsila Ferraz Frezza', \\ Maria Isabel Müller ${ }^{2}$ \& Karen Bazan Simionatto ${ }^{3}$ \\ ${ }^{1}$ Departamento de Biologia Animal, Instituto de Biologia, \\ Universidade Estadual de Campinas - UNICAMP, Cidade Universitária "Zeferino Vaz", \\ Rua Monteiro Lobato, 255, CEP 13083-862, Campinas, SP, Brasil \\ ${ }^{2}$ Instituto de Biologia, Curso de Pós-graduação em Parasitologia, \\ Universidade Estadual de Campinas - UNICAMP \\ ${ }^{3}$ Grupo de Pesquisas em Ecossistemas Aquáticos Sujeitos à Impactos Ambientais, \\ Faculdade de Ciências Biológicas, Pontifícia Universidade Católica - PUC, \\ Av. John Boyd Dunlop, s/n, Jd. Ipaussurama, CEP 13059-900, Campinas, SP, Brasil \\ ${ }^{4}$ Autor para correspondência: Rubens Riscala Madi,e-mail: rrmadi@gmail.com
}

MADI, R.R., UETA, M.T., FREZZA, T.F., MÜLLER, M.I. \& SIMIONATTO, K.B. Copepods Notodiaptomus sp. Kiefer (Crustacea, Calanoida) naturally infected by metacestodes in the Juqueri reservoir, São Paulo, Brazil. Biota Neotrop. 11(2): http://www.biotaneotropica.org.br/v11n2/en/abstract?article+bn03511022011

Abstract: The aim of this work was to identify the components of zooplankton that act as intermediate hosts of cestodes. One hundred and ninety four copepods of the suborder Calanoida, 317 copepods of the suborder Cyclopoida and 4240 cladocerans were collected in the Juqueri reservoir, in the state of São Paulo, from January to August, 2003. Only Copepods Calanoida of the genus Notodiaptomus sp. Kiefer were found to be infected and contained two distinct forms of metacestodes. The metacestodes, denominated Met 1 (order Proteocephalidea) and Met 2 (order Cyclophyllidea), had the following rates of prevalence and mean intensities of infection: Met 1 $2.06 \%$ and 64 larvae/copepod and Met $2-0.52 \%$ and one larvae/copepod. The positive copepods were collected at the margins of the reservoir during the day. This finding suggest that parasitism may lead to a change in the behavior of the copepods and make them more susceptible to predation in shallow water.

Keywords: copepoda, calanoida, metacestodes, cyclophyllidea, proteocephalidea.

MADI, R.R., UETA, M.T., FREZZA, T.F., MÜLLER, M.I. \& SIMIONATTO, K.B. Copépodos Notodiaptomus sp. Kiefer (Crustacea, Calanoida) naturalmente infectados com metacestódeos no reservatório do Juqueri, São Paulo, Brasil. Biota Neotrop. 11(2): http://www.biotaneotropica.org.br/v11n2/pt/abstract?article+bn03511022011

Resumo: Este trabalho teve o objetivo de identificar os componentes do zooplâncton que atuam como hospedeiros intermediários de cestódeos. Foram examinados 194 copépodos da subordem Calanoida, 317 copépodos da subordem Cyclopoida e 4240 cladóceros coletados no reservatório do Juqueri, Estado de São Paulo, entre janeiro e agosto de 2003. Apenas copépodos Calanoida do gênero Notodiaptomus sp. Kiefer encontravam-se positivos, sendo relatada duas formas morfológicas distintas de metacestódeos. Os metacestódeos, denominados Met 1 (Proteocephalidea) e Met 2 (Cyclophyllidea) apresentaram as seguintes prevalências e intensidades médias de infecção: Met 1 -2,06\% e 64 larvas/copépodo e Met 2-0,52\% e uma larva/copépodo. Os copépodos positivos foram coletados na região litorânea do reservatório durante o dia, o que leva a crer que o parasitismo pode modificar o comportamento dos copépodos tornando-os mais acessíveis à predação.

Palavras-chave: copepoda, calanoida, metacestódeos, cyclophyllidea, proteocephalidea. 


\section{Introdução}

O zooplâncton, parte da dieta de várias espécies de peixes, pode se tornar importante veiculador de parasitas atuando como hospedeiros intermediários de helmintos de peixes.

A ordem Cyclophyllidea van Beneden in Braun, 1900 é a maior ordem entre os cestódeos e todas as espécies são, em fase adulta, parasitas de vertebrados terrestres ou semi-aquáticos exceto peixes, onde se observa uma notável ausência. Podem, no entanto, se desenvolver em uma variada gama de hospedeiros intermediários invertebrados e vertebrados, incluindo peixes (Schmidt 1986). A ordem Proteocephalidea Mola, 1928 é encontrada apenas em animais relacionados com ambiente de água doce (peixes, anfíbios e répteis) (Rego 1994), utilizando, preferencialmente, copépodos como hospedeiros intermediários (Scholz 1999).

Após a ingestão, por copépodos, dos ovos de cestódeos ciclofilídeos e de proteocefalídeos disseminados na água pelo hospedeiro definitivo, a oncosfera eclode, atravessa ativamente a parede intestinal e aloja-se na hemocele do crustáceo. Dentro da cavidade, a oncosfera desenvolve a larva procercóide (proteocefalídeos) ou cisticercóide (ciclofilídeo). Em seguida, se o copépodo parasitado for ingerido por um peixe, no caso dos proteocefalídeos, a larva procercóide pode ainda penetrar pela parede intestinal, evoluindo para plerocercóide e então encapsular na cavidade peritoneal deste hospedeiro, somente se transformando em verme adulto após predação do peixe pelo hospedeiro definitivo (Olsen 1974, Scholz 1999).

Muito pouco se sabe sobre a dinâmica de transmissão dos parasitas aos peixes hospedeiros via zooplâncton (Marcogliese 1995). No Brasil a maioria dos trabalhos envolvendo zooplâncton de água doce restringe-se a levantamentos faunísticos, ecologia de populações e taxonomia/descrição de espécies, sendo raros estudos relacionando a biologia do zooplâncton com o parasitismo nos peixes (Pereira et al. 1936, Falavigna et al. 2000, 2003).

Esse trabalho teve por objetivo identificar os componentes da comunidade de microcrustáceos planctônicos, potenciais hospedeiros intermediários de cestódeos parasitas de peixes.

\section{Material e Métodos}

Os copépodos foram coletados no reservatório do Juqueri ( $23^{\circ} 19^{\prime} 07^{\prime}$ ' S, $46^{\circ} 35^{\prime} 12^{\prime \prime}$ O), bacia do Alto Tietê, região sudeste do Estado de São Paulo, entre janeiro e agosto de 2003. O reservatório é considerado eutrófico e possui $3,49 \mathrm{~km}^{2}$ de área, $28,77 \mathrm{~km}$ de perímetro e uma profundidade média de aproximadamente $12 \mathrm{~m}$. Inicia-se no centro urbano do Município de Mairiporã, estendendo-se por 9,5 km até ultrapassar o limite municipal com Franco da Rocha.

Na região limnética do reservatório os copépodos foram coletados por arrasto vertical, utilizando-se uma rede cônica de $90 \mathrm{~cm}$ de comprimento, $30 \mathrm{~cm}$ de diâmetro de abertura e malha de $154 \mu \mathrm{m}$, partindo-se de uma profundidade padrão de $20 \mathrm{~m}$, sendo o volume filtrado de aproximadamente 1400 L. Na região litorânea os copépodos foram coletados com auxílio de um balde de $20 \mathrm{~L}$, despejando-se a água através da rede cônica, perfazendo um total aproximado de $140 \mathrm{~L}$ filtrados. Das amostras finais concentradas em $250 \mathrm{ml}$, foi retirada uma alíquota de $25 \mathrm{ml}$ de cada amostra, e acrescentado $2 \mathrm{ml}$ de água carbonatada $7 \%$ para análise dos copépodos (Falavigna et al. 2003). Os indivíduos coletados foram colocados em placas de Petri, examinados ao microscópio estereoscópico e separados em grupos (Cladocera, Calanoida e Cyclopoida) e em seguida examinados ao microscópio óptico com uma fina camada de água sobre uma lâmina de vidro. Os exemplares que se apresentaram parasitados foram identificados e dissecados para isolamento e contagem dos parasitos.
Os cálculos da prevalência e intensidade de infecção foram realizados a partir das definições apresentadas por Bush et al. (1997).

\section{Resultados e Discussão}

Foram examinados no total 194 copépodos Calanoida (58 da região limnética e 136 da região litorânea), 317 copépodos Cyclopoida (165 da região limnética e 152 da região litorânea) e 4240 cladóceros (1319 da região limnética e 2921 da região litorânea). Foram encontradas duas formas morfológicas distintas de metacestódeos, denominadas Met 1 e Met 2, parasitando copépodos do gênero Notodiaptomus sp. (Calanoida) apenas nas amostras de água recolhidas na região litorânea do reservatório, nos meses de abril e maio (período de seca).

Os copépodos parasitados apresentaram taxas de prevalência de 2,06\% (4/194) para Met 1 e 0,52\% (1/194) para Met 2, totalizando $2,58 \%$ de copépodos infectados.

Os metacestódeos denominados Met 1 (Figura 1a,b), apresentavam forma oval, sem cercômero, mediam, em média, $70,98 \mu \mathrm{m}( \pm 4,33) \times 58,75 \mu \mathrm{m}( \pm 4,56)$, encontravam-se livres ocupando praticamente toda a hemocele. Não mostravam indícios de lacuna primária. O escólex, invaginado, apresentava quatro ventosas e mais uma ventosa apical, característico de cestódeos da ordem Proteocephalidea. A intensidade média de infecção foi 64 larvas/copépodo $( \pm 9,89)$.

O metacestódeo denominado Met 2 (Figura 1c,d) apresentou forma circular levemente ovalada, medindo $196,89 \times 189,67 \mu \mathrm{m}$, localizado entre o segundo e terceiro segmentos torácicos. O escólex, também invaginado, apresentou quatro ventosas e um conjunto de 20 acúleos medindo, em média, 26,11 $\mu \mathrm{m}$ de comprimento. Apenas um exemplar foi encontrado e foi identificado como pertencente à ordem Cyclophyllidea.

Marcogliese (1995) afirma que os copépodos tendem a apresentar taxas de prevalência de infecção por cestódeos variando entre $0,01 \%$ e $1,0 \%$. Falavigna et al. $(2000,2003)$ encontraram $0,3 \%$ de Thermocyclops minutus, Mesocyclops sp. e Paracyclops sp. infectados com um metacestódeo de proteocefalídeo cada, provável parasita de peixes encontrados na planície de inundação do rio Paraná. Hanzelová \& Gerdeaux (2003) registraram infecção natural de 0,21\% de Cyclops absorum por Proteocephallus longicollis no lago Annecy, França. Rusinek et al. (1996) encontraram 0,13\% de prevalência de Proteocephalus sp. em Epischura sp. (Calanoida) naturalmente infectados no lago Baikal, Russia. Marcogliese \& Esch (1989), também trabalhando com infecção natural, encontraram índices distintos de prevalência de Bothriocephalus acheilognathi parasitando Mesocyclops (0,6\%) e Tropocyclops (7,1\%), ambos no lago Belews, Estados Unidos. O valor da prevalência total encontrado neste trabalho $(2,58 \%)$ é superior aos valores encontrados pelos autores citados anteriormente.

Em hospedeiros intermediários invertebrados a manutenção e transmissão de cestódeos são efetivadas por poucos indivíduos dentro de uma população, isto é, ocorre distribuição agregada das formas infectantes (Roberts \& Janovy Junior 1996). Esse comportamento de agregação pode ser parte da estratégia de transmissão de Proteocephalidea, pois por não possuírem estágios de vida livre em seu ciclo (coracídio), esses cestódeos adotam a estratégia de recrutamento dos ovos na sua dispersão pela água (Esch 1983). Dupont \& Gabrion (1987) afirmam que as distribuições randômicas ou agregadas dos parasitas dependem também das variadas susceptibilidades apresentadas entre as espécies de copépodos hospedeiros. Em infecção experimental Wedekind (1997) demonstra que a probabilidade de um copépodo se tornar infectado aumenta com o crescimento do número de parasitas administrados. A intensidade 

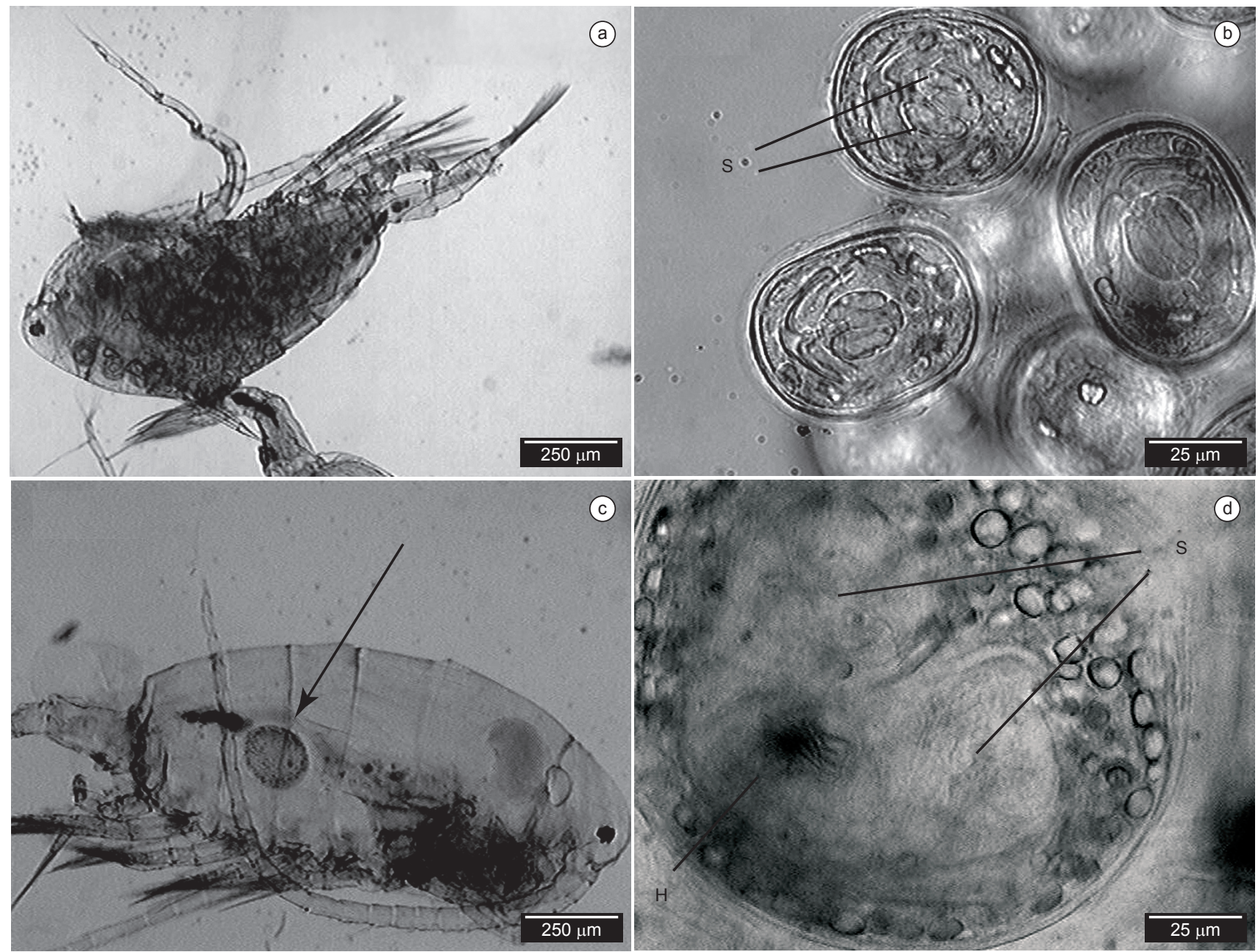

Figura 1. Notodiaptomus sp. parasitados por metacestódeos Met 1 (a, b) e Met 2 (c, d). H - ganchos; S - ventosas.

Figure 1. Notodiaptomus sp. parasitized by metacestodes Met 1 (a, b) and Met 2 (c, d). H - hooks; S - suckers.

de infecção encontrada em copépodos parasitados por metacestódeos Proteocephalidea neste trabalho corrobora a hipótese de distribuição agregada entre os cestódeos dessa ordem.

Van der Veen \& Kurtz (2002) observaram que durante a fase de desenvolvimento dos metacestódeos, alguns exemplares de parasitas morrem e que copépodos imaturos apresentam uma maior tendência a infecção, mas morrem mais facilmente que copépodos adultos. Van der Veen (2003) afirma ainda que os copépodos imaturos apresentam maior atividade de locomoção e ingerem mais parasitas que copépodos adultos. O aumento da mortalidade de copépodos após três semanas de infecção encontrado por Pasternak et al. (1999), pode corroborar a explicação do paradoxo das relativas baixas taxas de prevalência do primeiro hospedeiro intermediário - copépodo - em relação às altas taxas encontradas no segundo hospedeiro do ciclo - peixe, uma vez que Madi \& Ueta (2002) trabalhando neste mesmo reservatório encontraram, $79,91 \%$ de Geophagus brasiliensis - Cichlidae - infectados com larvas plerocercóides de proteocefalídeos.

Alguns helmintos podem provocar alterações comportamentais de seus hospedeiros, fazendo com que os copépodos infectados se agreguem e/ou tenham dificuldade de natação, facilitando assim sua captura pelos peixes hospedeiros (Pulkkinen et al. 2000). As dificuldades de escape ao predador impostas pelo parasita ao copépodo hospedeiro, podem ser associadas aos efeitos do parasita sobre o sistema nervoso do copépodo infectado, via neurotransmissores, fazendo com que diminua o desempenho muscular e aumente a atividade de busca por alimentos (Franz \& Kurtz 2002). Alguns copépodos apresentam ainda fotofilia quando parasitados por larvas de cestódeos (Pulkkinen et al. 2000). Essas alterações no comportamento podem explicar o encontro de copépodos infectados na região litorânea do reservatório durante o dia, uma vez que trabalhos sobre migração vertical do zooplâncton descrevem que nesse período copépodos calanoidas estão em locais mais profundos do reservatório (Esteves 1998).

\section{Agradecimentos}

À Silvia Maria Caglierani Casanova do Departamento de Zoologia, IBB, Unesp, pela identificação dos copépodos, à Sabesp pelo apoio em campo. À Maurício Solera Rodrigues da Silva e João Batista Alves de Oliveira, pelo auxílio em laboratório e em campo.

\section{Referências Bibliográficas}

BUSH, A.O., LAFFERTY, K.D., LOTZ, J.M. \& SHOSTAK, A.W 1997. Parasitology meet ecology on its own terms: Margolis et al. revisited. J. Parasitol. 83(4):575-583. PMid:9267395. http://dx.doi. org/10.2307/3284227

DUPONT, F. \& GABRION, C. 1987. The concept of specificity in the procercoid-copepod system: Bothrocephalus claviceps (Cestoda) a parasite of the eel (Anguilla anguilla). Parasitol. Res. 73(2):151-158. PMid:3575289. http://dx.doi.org/10.1007/BF00536472 
ESCH, G.W. 1983. The population and community ecology of cestodes. In Biology of Eucestoda (C. Arme \& P.W. Pappas eds.). Academic Press, Londres, v.1, p.81-137.

ESTEVES, F.A. 1998. Fundamentos de Limnologia. 2nd ed. Interciência, Rio de Janeiro.

FALAVIGNA, D.L.M., PAVANELLI, G.C. \& TAKEMOTO, R. 2000. Resultados preliminares do ciclo evolutivo de cestóides parasitas de Pseudoplatystoma corruscans da planície de inundação do alto do Rio Paraná, Brasil. In VI Encontro Brasileiro de Patologistas de Organismos Aquáticos. Universidade Federal de Santa Catarina e ABRAPOA, Florianópolis, p.133.

FALAVIGNA, D.L.M., VELHO, L.F.M. \& PAVANELLI, G.C. 2003. Proteocephalidean larvae (Cestoda) in naturally infected Cyclopid copepods of the upper Paraná river floodplain, Brazil. Mem. Inst. Oswaldo Cruz. 98(1):69-72. http://dx.doi.org/10.1590/S0074-02762003000100009

FRANZ, K. \& KURTZ, J. 2002. Altered host behaviour: manipulation or energy depletion in tapeworm-infected copepods? Parasitology 125(2):187-196. http://dx.doi.org/10.1017/S0031182002001932

HANZELOVÁ, V. \& GERDEAUX, D. 2003. Seasonal ocurrence of the tapeworm Proteocephalus longicollis and its transmission from copepod intermediate host to fish. Parasitol. Res. 91(2):130-136. PMid:12910414. http://dx.doi.org/10.1007/s00436-003-0939-x

MADI, R.R. \& UETA, M.T. 2002. Estudo comparativo dos metazoários parasitas de Geophagus brasiliensis (Cichlidae, Perciforme) em dois reservatórios no estado de São Paulo. In VII Encontro Brasileiro de Patologistas de Organismos Aquáticos. UEM, Foz do Iguaçu, p.137.

MARCOGLIESE, D.J. 1995. The role of zooplankton in the transmission of helminth parasites to fish. Rev. Fish Biol. Fisher. 5:336-371. http://dx.doi. org/10.1007/BF00043006

MARCOGLIESE, D.J. \& ESCH, G.W. 1989. Experimental and natural infection of planktonic anb benthic copepods by the asian tapeworm, Bothriocephalus acheilognathi. Proc. Helminthol. Soc. Wash. 56(2):151-155.

OLSEN, O.W. 1974. Animal parasites. Their life cicles and ecology. Univ. Park Press, Baltimore.
PASTERNAK, A. F., PULKKINEN, K., MIKHEEV, V.N., HASU, T. \& VALTONEN, E.T. 1999. Factors affecting abundance of Triaenophorus infection in Cyclops strenuus, and parasite-induced changes in host fitness. Int. J. Parasitol. 29(11):1793-1801. http://dx.doi.org/10.1016/ S0020-7519(99)00108-3

PEREIRA, C., VIANNA DIAS, M. \& AZEVEDO, P. 1936. Biologia do nematóide Procamallanus cearensis n. sp. Arch. Inst. Biológico 7:209-226.

PULKKINEN, K., PASTERNAK, A.F., HASU, T. \& VALTONEN, E.T. 2000. Effect of Triaenophorus crassus (Cestoda) infection on behavior and susceptibility to predation of the first intermediate host Cyclops strenuus (Copepoda). J. Parasitol. 86(4):664-670.

REGO, A. A. 1994. Order Protocephalidea Mola, 1928. In Keys to the Cestode Parasites of Vertebrates. (L.F. Khalil, A. Jones, \& R.A. Bray eds.). CAB International, Wallingford, p.257-293.

ROBERTS, L.S. \& JANOVY JUNIOR, J. 1996. Foundations of Parasitology. 5nd ed. WCB Publishers, Dubuque.

RUSINEK, O.T., BAKINA, M.P. \& NIKOLSKII, A.V. 1996. Natural infection of the calanoid crustacean Epischura baicalensis by procercoids of Proteocephalus sp. in Listvenichnyi Bay, Lake Baikal. J. Helminthol. 70(3):237-247. http://dx.doi.org/10.1017/S0022149X00015479

SCHMIDT, G.D. 1986. Handbook of tapeworm identification. CRC Press, Boca Raton.

SCHOLZ, T. 1999. The life cycles of species of Proteocephalus, parasites of fishes in Palearctic Region: a review. J. Helminthol. 73(1):1-19.

Van der VEEN, I.T. 2003. Is body size or activity of copepods related to ingestion of parasite larvae? Parasitology 126(2):173-178. PMid:12636355. http://dx.doi.org/10.1017/S0031182002002652

Van der VEEN, I.T. \& KURTZ, J. 2002. To avoid or eliminate: cestode infections in copepods. Parasitology 124(4):465-474. PMid:12003070. http://dx.doi.org/10.1017/S0031182001001275

WEDEKIND, C. 1997. The infectivity, growth, and virulence of the cestode Schistocephalus solidus in its first intermediate host, the copepod Macrocyclops albidus. Parasitology 115(3):317-324. PMid:9300470. http://dx.doi.org/10.1017/S0031182097001406

Recebido em 17/03/2010

Versão reformulada recebida em 30/05/2011

Publicado em 17/06/2011 